\title{
Libration Points Inside a Spherical Cavity of a Uniformly Rotating Gravitating Ball
}

\author{
A. A. Burov, V. I. Nikonov
}

Dedicated to the memory of A. V. Borisov

The problem of the existence and stability of relative equilibria (libration points) of a uniformly rotating gravitating body, which is a homogeneous ball with a spherical cavity, is considered. It is assumed that the rotation is carried out around an axis perpendicular to the axis of symmetry of the body and passing through its center of mass. The libration points located inside the cavity are investigated. Families of both isolated and nonisolated relative equilibria are found. Their stability and bifurcations are investigated. Realms of possible motion are constructed.

Keywords: celestial bodies with cavities, libration points, relative equilibria, motion in a noncentral gravitational field, gravitating dumbbell

\section{Introduction}

The study of motion in the field of attraction of celestial bodies still remains a problem that attracts special attention. The interest in it, in particular, is supported by the need to study the range of problems aimed at stopping Asteroid-Comet Hazard (see, e. g., [1-4]). At the same time, the problems associated with the motion inside the cavities that celestial bodies may possess remain somewhat in the shadow. The present work is aimed at filling this gap, in particular, at demonstrating some rather unexpected dynamic properties observed in such problems.

Received July 28, 2021

Accepted October 29, 2021

The research is supported by Russian Science Foundation (project No. 22-21-00297)

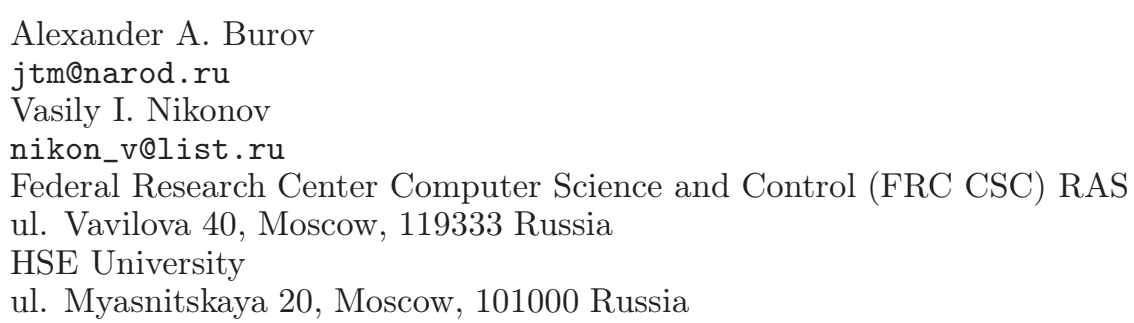

RUSSIAN JOURNAL OF NONLINEAR DYNAMICS, 2021, 17(4), 413-427 


\section{Problem statement and basic notation}

Consider a body $\mathcal{A}$ obtained from a homogeneous ball $\mathcal{B}$ with the center $B$ of radius $r_{B}$ by removing the contents of a spherical cavity $\mathcal{C}$ with the center $C$ of radius $r_{C}: \mathcal{A}=\mathcal{B} \backslash \mathcal{C}$. Let the point $O$ be the center of mass of the body $\mathcal{A}$. Suppose that this body performs a uniform rotation with a constant angular velocity $\omega$ around an axis perpendicular to the axis $B C$ and passing through the point $O$. Let the material point (particle) $P$ move in the gravitational field of the body $\mathcal{A}$. In what follows, we assume that the particle is negligibly small compared to the body and does not affect its motion.

We introduce a rectangular frame of reference $O x_{1} x_{2} x_{3}$ rotating together with the body (RF), the axis $O x_{3}$ of which coincides with the axis of rotation, the axis $O x_{1}$ is directed along the axis of symmetry of the body, and the axis $\mathrm{Ox}_{2}$ complements them to the right triple (Fig. 1). The unit vectors of the axes of the RF are denoted by $\mathbf{e}_{1}, \mathbf{e}_{2}$ and $\mathbf{e}_{3}$, respectively.

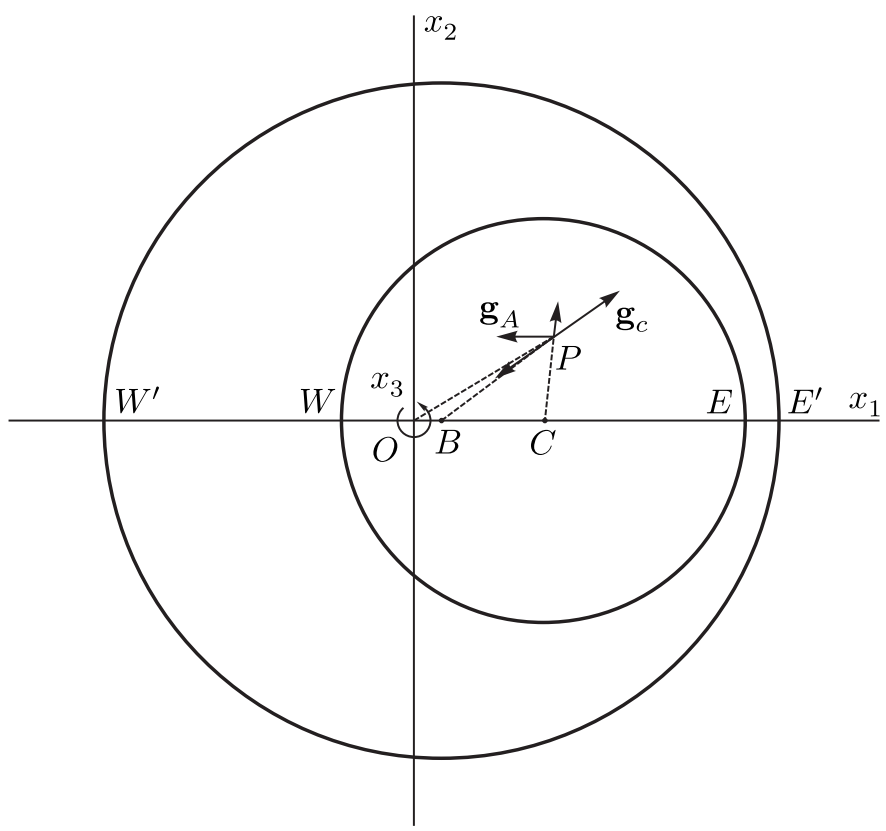

Fig. 1

We intend to study the equilibria of the point $P$ with respect to the rotating frame. In celestial mechanics, such equilibria are usually called libration points.

Let the center of the cavity $C$ be located on the positive semi-axis $O x_{1}$. Further, it is assumed that the cavity $\mathcal{C}$ does not extend beyond the outer surface of the body, i. e., $d+$ $+r_{C} \leqslant r_{B}$. If $|B C|=d$, then

$$
\begin{gathered}
\mathbf{O P}=\mathbf{r}=\left(x_{1}, x_{2}, x_{3}\right)^{T}, \quad \text { OB }=(b, 0,0)^{T}, \quad \text { OC }=(c, 0,0)^{T}, \\
b=\frac{r_{C}^{3}}{r_{B}^{3}-r_{C}^{3}} d, \quad c=\frac{r_{B}^{3}}{r_{B}^{3}-r_{C}^{3}} d>0 .
\end{gathered}
$$

The point $P$ moves relative to the noninertial frame of reference $O x_{1} x_{2} x_{3}$ under the influence of the force of attraction from the body, the centrifugal and Coriolis forces. In the case of contact of the point $P$ with the internal or external boundaries of the body $\partial \mathcal{A}=\partial \mathcal{B} \cup \partial \mathcal{C}$, its motion is also affected by normal reaction and, under appropriate assumptions, by friction force. 
In the absence of contact with the surface, the equations of motion read

$$
\begin{gathered}
\frac{d}{d t} \frac{\partial L}{\partial \mathbf{v}}=\frac{\partial L}{\partial \mathbf{r}}, \quad \dot{\mathbf{r}}=\mathbf{v}, \\
L=\frac{1}{2}\left(\mathbf{v}+\omega \mathbf{e}_{3} \times \mathbf{r}, \mathbf{v}+\omega \mathbf{e}_{3} \times \mathbf{r}\right)-U(\mathbf{r}) .
\end{gathered}
$$

Here $\mathbf{v}=\left(v_{1}, v_{2}, v_{3}\right)^{T}$ is the velocity vector of the point $P$ relative to the $R F, U=U(\mathbf{r})$ is the potential of the attractive forces. The equations are reduced to the form

$$
\frac{d \mathbf{v}}{d t}=2 \omega \mathbf{v} \times \mathbf{e}_{3}+\mathbf{g}_{c}+\mathbf{g}_{A}, \quad \dot{\mathbf{r}}=\mathbf{v} ; \quad \mathbf{g}_{c}=-\frac{\partial U_{c}}{\partial \mathbf{r}}, \quad \mathbf{g}_{A}=-\frac{\partial U}{\partial \mathbf{r}} .
$$

Here $\mathbf{g}_{c}$ is the intensity of centrifugal forces with the potential

$$
U_{c}=-\frac{1}{2} \omega^{2}\left(\mathbf{e}_{3} \times \mathbf{r}, \mathbf{e}_{3} \times \mathbf{r}\right),
$$

and $\mathbf{g}_{A}$ is the intensity of the field of attraction (Fig. 1). In the case where the point $P$ touches the surface, the equations of motion have the form

$$
\frac{d \mathbf{v}}{d t}=2 \omega \mathbf{v} \times \mathbf{e}_{3}+\mathbf{g}_{c}+\mathbf{g}_{A}+\mathbf{g}_{N}+\mathbf{g}_{T}, \quad \dot{\mathbf{r}}=\mathbf{v},
$$

where $\mathbf{g}_{N}$ and $\mathbf{g}_{T}$ are the normal reaction of the surface and the friction force related to the mass of the point.

One can single out three cases that differ significantly from the point of view of dynamics:

- the point $P$ is located inside the cavity $\mathcal{C}$,

- the point $P$ is outside the ball $\mathcal{B}$,

- the point $P$ is located inside the body $\mathcal{A}$, assumed to be permeable ("Robe's problem" [5]).

Each of these cases is interesting in its own right. In this paper, the focus is on the case where the point $P$ is located inside the cavity $\mathcal{C}$ or on its surface $\partial \mathcal{C}$. Let us also assume that there is no friction.

\section{Structure of the field of attraction}

As is well known (see, e.g., [6], problem O-130), inside the cavity, the intensity of the field of attraction from the side of the body $\mathcal{A}$ has the form

$$
\mathbf{g}_{A}=-\frac{4}{3} \pi G \rho \mathbf{e}_{1}=-\frac{4}{3} \pi G \rho d(1,0,0)^{T},
$$

where $G$ is the gravitational constant and $\rho$ is the density of the body $\mathcal{A}$. In other words, the field of attraction forces inside the cavity is constant and uniform. Inside the body, but outside the cavity, the intensity of the field of attraction reads

$$
\mathbf{g}_{A}=-\frac{4}{3} \pi G \rho\left(\mathbf{B P}-\frac{r_{C}^{3}}{|\mathbf{C P}|^{3}} \mathbf{C P}\right) .
$$

Finally, outside the body, the intensity of the field of attraction has the form

$$
\mathbf{g}_{A}=-\frac{4}{3} \pi G \rho\left(\frac{r_{B}^{3}}{|\mathbf{B P}|^{3}} \mathbf{B P}-\frac{r_{C}^{3}}{|\mathbf{C P}|^{3}} \mathbf{C P}\right) .
$$


The force of attraction is potential, its potential is written as

$$
U=\frac{4}{3} \pi G \rho \cdot \begin{cases}d \cdot x_{1}, & P \in \mathcal{C}, \\ \frac{1}{2}\left(|\mathbf{B P}|^{2}-3 r_{B}^{2}\right)+\frac{r_{C}^{3}}{|\mathbf{C P}|}, & P \in \mathcal{B} \backslash \mathcal{C}, \\ -\frac{r_{B}^{3}}{|\mathbf{B P}|}+\frac{r_{C}^{3}}{|\mathbf{C P}|}, & P \in \mathbb{R}^{3} \backslash \mathcal{B} .\end{cases}
$$

REMARK 1. In the case where the point $P$ is located inside the cavity, equations (2.1) are linear and therefore integrable in the final form.

REMARK 2. The principal central moments of inertia of the body $\mathcal{A}$ can be determined by the Huygens - Steiner theorem (see, e.g., [7])

$$
\begin{gathered}
I_{1}^{\mathcal{A}}(O)=I_{1}^{\mathcal{B}}(O)-I_{1}^{\mathcal{C}}(O)=I_{1}^{\mathcal{B}}(B)-I_{1}^{\mathcal{C}}(C) \\
I_{2}^{\mathcal{A}}(O)=I_{3}^{\mathcal{A}}(O)=I_{3}^{\mathcal{B}}(O)-I_{3}^{\mathcal{C}}(O)=I_{3}^{\mathcal{B}}(B)+m_{B} b^{2}-\left(I_{3}^{\mathcal{C}}(C)+m_{C} c^{2}\right) .
\end{gathered}
$$

Explicit expressions for these moments of inertia in the problem parameters have the form

$$
I_{1}^{\mathcal{A}}(O)=\frac{8}{15} \rho \pi\left(r_{B}^{5}-r_{C}^{5}\right), \quad I_{2}^{\mathcal{A}}(O)=I_{3}^{\mathcal{A}}(O)=\frac{8}{15} \rho \pi\left(r_{B}^{5}-r_{C}^{5}\right)-\frac{4}{3} \rho \pi\left(r_{B}^{3}-r_{C}^{3}\right) \cdot b \cdot c .
$$

It can be seen from relations (3.1) that the moment of inertia $I_{1}^{\mathcal{A}}(O)$ relative to the axis of symmetry of the body is the largest.

\section{Existence of libration points}

To determine the libration points, the critical points of the augmented potential [8-11]

$$
U_{a}=U_{c}+U
$$

are studied. If the area of the possible location of libration points has boundaries, then the Routh method is used. As is well known, this method reduces to the method of Lagrange multipliers. In the case where the particle $P$ is located inside the cavity $\mathcal{C}$, the unilateral constraint is given by the inequality

$$
f=\frac{1}{2}\left(\left(x_{1}-c\right)^{2}+x_{2}^{2}+x_{3}^{2}-r_{C}^{2}\right) \leqslant 0
$$

Let's introduce a dimensionless angular velocity $\Omega \geqslant 0: \Omega^{2}=\frac{3}{4} \frac{\omega^{2}}{\pi G \rho}$ and take the radius of the ball $\mathcal{B}$ as a unit of length: $r_{B}=1$. Let

$$
\mathcal{W}=U_{a}+\frac{4}{3} \pi G \rho \lambda f
$$

be the Routh function, where $\lambda$ is an indefinite Lagrange multiplier.

The equations of the critical points of function (4.1) under condition (4.2) have the form

$$
0=\frac{\partial \mathcal{W}}{\partial x_{1}}=-\Omega^{2} x_{1}+d+\lambda\left(x_{1}-c\right), \quad 0=\frac{\partial \mathcal{W}}{\partial x_{2}}=\left(\lambda-\Omega^{2}\right) x_{2}, \quad 0=\frac{\partial \mathcal{W}}{\partial x_{3}}=\lambda x_{3} .
$$

System (4.3) should be considered together with inequality (4.2), taking into account the fact that constraint (4.2) is unilateral, and the inequality $\lambda \geqslant 0$ should be fulfilled on the solutions under consideration. 
System (4.3) has four types of solutions:

\begin{tabular}{|c|l|lc|c|}
\hline$I_{W}:$ & $\lambda=\lambda_{W}$ & $x_{1}=c-r_{C}$, & $x_{2}=0$ & $x_{3}=0$ \\
\hline$I_{E}:$ & $\lambda=\lambda_{E}$ & $x_{1}=c+r_{C}$, & $x_{2}=0$ & $x_{3}=0$ \\
\hline$I_{C}:$ & $\lambda=\lambda_{C}$ & $\left(x_{1}, x_{2}\right):$ & $\left(x_{1}-c\right)^{2}+x_{2}^{2}=r_{C}^{2}$ & $x_{3}=0$ \\
\hline$I_{L}:$ & $\lambda=0$ & $x_{1}=x_{1 \star}$, & $x_{2}=0$ & $-x_{3 *} \leqslant x_{3} \leqslant x_{3 *}$ \\
\hline
\end{tabular}

Here

$$
\begin{gathered}
x_{1 \star}=\frac{d}{\Omega^{2}}, \quad x_{3 *}=\sqrt{r_{C}^{2}-d^{2}\left(\frac{1}{\Omega^{2}}-\frac{1}{1-r_{C}^{2}}\right)^{2}}, \\
\lambda_{W}=\left(c-r_{C}\right) \frac{\Omega_{\star \star}^{2}-\Omega^{2}}{r_{C}}, \quad \lambda_{E}=\left(c+r_{C}\right) \frac{\Omega^{2}-\Omega_{\star}^{2}}{r_{C}}, \quad \lambda_{C}=\Omega_{0}^{2}, \\
\Omega_{\star}^{2}=\left(\frac{1}{1-r_{C}^{3}}+\frac{r_{C}}{d}\right)^{-1}=\frac{d}{c+r_{C}}, \quad \Omega_{0}^{2}=\frac{d}{c}, \quad \Omega_{\star \star}^{2}=\left(\frac{1}{1-r_{C}^{3}}-\frac{r_{C}}{d}\right)^{-1}=\frac{d}{c-r_{C}} .
\end{gathered}
$$

The existence of libration points forming families $I_{W}$ and $I_{E}$ and parametrized by angular velocity is quite predictable. The existence of nonisolated libration points forming families $I_{C}$ and $I_{L}$ is surprising at first glance. Nonisolated libration points $I_{C}$ that fill a circle in the plane $O x_{1} x_{2}$ exist at a single value of the angular velocity. Nonisolated libration points $I_{L}$ exist at the values of the angular velocity belonging, generally speaking, to a certain segment. These libration points are also nonisolated, and for each permissible value of the angular velocity they occupy a segment parallel to the axis of rotation. The existence of these points has turned out to be also unexpected.

\section{Parametric analysis of libration points}

There are three cases that differ from each other by dynamic properties:

a) the center of mass $O$ is located inside the cavity $\mathcal{C}$, i. e., $c<r_{C}$,

b) the center of mass $O$ is located on the boundary of the cavity $\partial \mathcal{C}$, i. e., $c=r_{C}$,

c) the center of mass $O$ does not belong to the cavity $\mathcal{C}$, i. e., $c>r_{C}$.

On the parameter plane $\left(r_{C}, d\right)$, physically meaningful values correspond to points inside an isosceles right triangle $A$ with a hypotenuse $d+r_{C}=1$. The curve $\Gamma$, given by the equation

$$
d=r_{C}\left(1-r_{C}^{3}\right)
$$

divides this triangle into parts $A_{1}$ and $A_{2}$. For points from $A_{1}$, the condition $c<r_{C}$ is satisfied. For the points belonging to the curve $\Gamma$, the condition $c=r_{C}$ is satisfied. Finally, the condition $c>r_{C}$ is satisfied for the points from $A_{2}$ (Fig. 2). Let us consider these cases separately, paying special attention to the condition of nonnegativity of the constraint reaction.

For the $I_{W}$ libration points, the reaction is positive if the condition

$$
d>\Omega^{2}\left(c-r_{C}\right)
$$

is satisfied. For $c \leqslant r_{C}$, this condition is satisfied for all values of $\Omega$, while for $c>r_{C}$, this condition takes the form

$$
\Omega^{2}<\Omega_{\star \star}^{2} .
$$




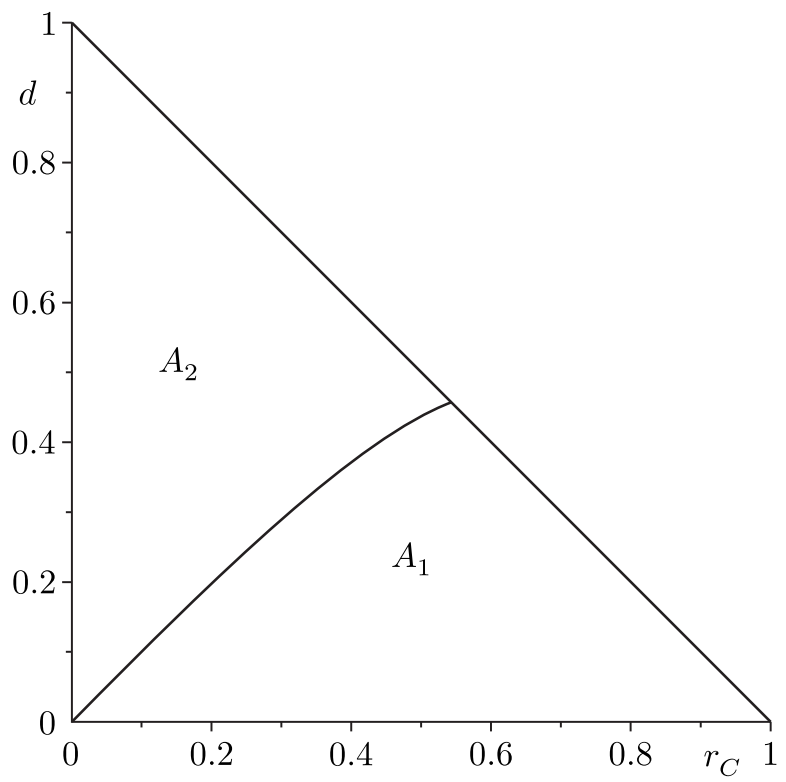

Fig. 2. The plane of independent parameters $\left(r_{C}, d\right)$

For the $I_{E}$ libration points, the reaction is positive if the condition

$$
d>\Omega^{2}\left(c+r_{C}\right) \Longleftrightarrow \Omega^{2}>\Omega_{\star}^{2}
$$

is satisfied.

For the $I_{C}$ libration points, the reaction is always positive.

For the $I_{L}$ family the libration points fill the chord parallel to the axis $O x_{3}$ and passing through the point $\left(x_{1 \star}, 0,0\right)$. The value $x_{3 \star}$ determines the ends of this chord. In addition, the inequality

$$
\left|c-x_{1 \star}\right| \leqslant r_{C}
$$

corresponding to the location of libration points inside the cavity $\mathcal{C}$, is reduced to the form

$$
\left|\frac{d}{1-r_{C}^{3}}-\frac{d}{\Omega^{2}}\right| \leqslant r_{C} \Longleftrightarrow-\frac{r_{C}}{d} \leqslant \frac{1}{1-r_{C}^{3}}-\frac{1}{\Omega^{2}} \leqslant \frac{r_{C}}{d} .
$$

If the condition

$$
c \leqslant r_{C} \Longleftrightarrow \frac{1}{1-r_{C}^{3}}-\frac{r_{C}}{d} \leqslant 0
$$

is satisfied, then the solution of inequality (5.1) has the form

$$
\Omega_{\star}^{2} \leqslant \Omega^{2} .
$$

Otherwise, when $c>r_{C}$, the solution of inequality (5.1) has the form

$$
\Omega_{\star}^{2} \leqslant \Omega^{2} \leqslant \Omega_{\star \star}^{2} .
$$

Inequalities (5.2) and (5.3) impose restrictions on the value of angular velocity at which the $I_{L}$ libration points exist. At the same time, if $c \rightarrow r_{C}$, then $\Omega_{\star \star} \rightarrow \infty$.

The constraint is released on the $I_{L}$ libration points, i. e., the magnitude of its reaction is equal to zero.

REMARK 3. When $\Omega=\Omega_{\star}$, the $I_{L}$ family of libration points branches off from the $I_{E}$ family. For $\Omega=\Omega_{\star \star}$, the $I_{L}$ family of libration points is connected to the $I_{W}$ family for $c>r_{C}$. For $c \leqslant r_{C}$, the libration points from the $I_{L}$ family tend to the axis of rotation with an unlimited increase of the angular velocity. 


\section{Stability of libration points and bifurcation diagrams}

To study sufficient stability conditions according to the Routh method [8,9] (see also [10, $11]$ ), consider the sign-definiteness of the second variation of the augmented potential, restricted to a linear manifold determined by the constraint, if it is imposed.

\subsection{Libration points $I_{L}$}

There are no constraints for solutions of $I_{L}$, and the expression for the second variation has the form

$$
\begin{gathered}
2 \delta^{2} U=\left(\frac{\partial^{2} U}{\partial \mathbf{r}^{2}} \delta \mathbf{r}, \delta \mathbf{r}\right)=\sigma_{1} \delta x_{1}{ }^{2}+\sigma_{2} \delta x_{2}{ }^{2}+\sigma_{3} \delta x_{3}{ }^{2}, \\
\delta \mathbf{r}=\left(\delta x_{1}, \delta x_{2}, \delta x_{3}\right)^{T}, \quad \sigma_{1}=\sigma_{2}=-\Omega^{2}, \quad \sigma_{3}=0 .
\end{gathered}
$$

Two Poincaré coefficients are negative, the third one is equal to zero. Thus, libration points from the $I_{L}$ family are unstable.

\subsection{Libration points $I_{W}, I_{E}$ and $I_{C}$}

The $I_{W}, I_{E}$ and $I_{C}$ libration points are located on the surface of the cavity. Therefore, when studying stability, it is necessary to take into account the fact that these libration points exist due to the presence of a unilateral constraint. For $I_{W}, I_{E}$ and $I_{C}$ libration points, the expressions for linear manifolds and second variations are as follows:

$$
\begin{aligned}
I_{W}: \delta x_{1}=0, & 2 \delta^{2} \mathcal{W}=c \frac{\Omega_{0}^{2}-\Omega^{2}}{r_{C}} \delta x_{2}^{2}+\left(c-r_{C}\right) \frac{\Omega_{\star \star}^{2}-\Omega^{2}}{r_{C}} \delta x_{3}^{2} ; \\
I_{E}: \delta x_{1}=0, & 2 \delta^{2} \mathcal{W}=c \frac{\Omega^{2}-\Omega_{0}^{2}}{r_{C}} \delta x_{2}^{2}+\left(c+r_{C}\right) \frac{\Omega^{2}-\Omega_{\star}^{2}}{r_{C}} \delta x_{3}^{2} ; \\
I_{C}:\left(x_{0}-c\right) \delta x_{1}+y_{0} \delta x_{2}=0, & 2 \delta^{2} \mathcal{W}=\Omega_{0}^{2} \delta x_{3}{ }^{2},
\end{aligned}
$$

respectively. Calculations show that the stability properties of solutions and the structure of bifurcation diagrams depend on whether the inequality $c>R_{C}$ is satisfied or not.

\subsubsection{The case $c>r_{C}$}

At $\Omega=0$, the libration point from $I_{W}$ is stable in the sense of Lyapunov: the degree of instability is zero. The $I_{W}$ libration points remain stable at $0 \leqslant \Omega<\Omega_{0}$. For $\Omega=\Omega_{0}$ the libration point from $I_{W}$ becomes unstable with a degree of instability equal to one. The loss of stability is accompanied by branching off the $I_{C}$ family of nonisolated libration points from the $I_{W}$ family. As long as the inequality $\Omega_{0}<\Omega<\Omega_{\star \star}$ remains satisfied, the $I_{W}$ libration points remain unstable with the degree of instability $\chi=1$. When $\Omega=\Omega_{\star \star}$ a branch of the $I_{L}$ family of nonisolated libration points meets the family $I_{W}$. If the condition $\Omega>\Omega_{\star \star}$ is satisfied, then the $I_{W}$ family no longer exists due to releasing of the unilateral constraint. Along with the $I_{W}$ libration points, the $I_{L}$ libration points also disappear.

At $0 \leqslant \Omega<\Omega_{\star}$, the $I_{E}$ libration points do not exist, since the unilateral constraint is not strained. For $\Omega=\Omega_{\star}$, both families $I_{E}$ and $I_{L}$ of libration points appear from one point of the bifurcation diagram. While the inequality $\Omega_{\star}<\Omega<\Omega_{0}$ is fulfilled, the degree of instability of $I_{E}$ libration points is equal to one. For $\Omega=\Omega_{0}$, nonisolated libration points $I_{C}$ branch off from the $I_{E}$ family, and for $\Omega>\Omega_{0}$, the $I_{E}$ libration points are stable in the sense of Lyapunov: the degree of instability is zero.

In conclusion, we note that for the $I_{C}$ libration points, one of the Poincaré coefficients vanishes, while the second is always positive. 


\subsubsection{The case $c \leqslant r_{C}$}

At $\Omega=0$, the libration point from the $I_{W}$ family is stable in the sense of Lyapunov: the degree of instability is zero. The $I_{W}$ libration points remain stable at $0 \leqslant \Omega<\Omega_{0}$. For $\Omega=\Omega_{0}$, a family $I_{C}$ of nonisolated libration points branches off from the $I_{W}$ family, and for $\Omega>\Omega_{0}$, the $I_{W}$ libration points are unstable with the degree of instability equal to one.

At $0 \leqslant \Omega<\Omega_{\star}$, the $I_{E}$ libration points do not exist, since the unilateral constraint is not strained. For $\Omega=\Omega_{\star}$, both $I_{E}$ and $I_{L}$ families of libration points appear from one point of the bifurcation diagram. In this case, the degree of instability of the $I_{E}$ libration points is $\chi=1$. For $\Omega=\Omega_{0}$, the $I_{C}$ nonisolated libration points branch off from the $I_{E}$ family, and for $\Omega>\Omega_{0}$, the $I_{E}$ libration points are stable in the sense of Lyapunov. For $\Omega \rightarrow \infty$, libration points from the $I_{L}$ family tend to the axis of rotation.

In conclusion, we note again that for the $I_{C}$ libration points, one of the Poincaré coefficients vanishes, while the second is always positive.

REMARK 4. Since it is assumed that the system under consideration is subjected to the unilateral constraint, the results of [12] could be used in the study of stability (see also [13]). Let us confine ourselves to the remark that for those relative equilibria for which the degree of instability is proved to be zero, stability along the radius is provided by the positivity of the normal reaction.

\section{Bifurcation diagrams and realms of possible motion}

The realm of possible motion (RPM) is the intersection of an region $\mathcal{S}$ in a three-dimensional space determined by inequality

$$
\mathcal{S}: \quad U-h \leqslant 0, \quad U=-\frac{\Omega^{2}\left(x_{1}^{2}+x_{2}^{2}\right)}{2}+d x_{1},
$$

with the cavity $\mathcal{C}$ defined by inequality (4.2).

Let $\Omega=0$. In this simplest case the region $\mathcal{S}$ is a half-space determined by the inequality

$$
d x_{1} \leqslant h .
$$

If $h<d\left(c-r_{C}\right)$, then the RPM is empty. If $h=d\left(c-r_{C}\right)$, then the RPM consists of a single point $W$, which is a stable libration point. If $d\left(c-r_{C}\right)<h<d\left(c+r_{C}\right)$, then the RPM is a spherical segment of the cavity $\mathcal{C}$ adjacent to the point $W$. If $h \geqslant d\left(c+r_{C}\right)$, then the RPM is the entire cavity of $\mathcal{C}$, i. e., the ball.

Now let us assume $\Omega>0$. After transformations inequality (7.1) is reduced to the form

$$
\left(x_{1}-d \Omega^{-2}\right)^{2}+x_{2}^{2} \geqslant \Omega^{-4}\left(d^{2}-2 \Omega^{2} h\right) .
$$

It defines a straight circular cylinder in three-dimensional Euclidean space. Its axis is parallel to the axis $\mathrm{Ox}_{3}$. The further goal is to determine the relative position of this cylinder and the cavity depending on the values of the parameters.

For $d^{2}-2 \Omega^{2} h<0$, inequality (7.2) holds true in the whole space without a boundary, i. e., the RPM is the ball $\mathcal{C}$.

For $d^{2}-2 \Omega^{2} h=0$, inequality (7.2) holds true in the whole space with a boundary consisting of a straight line

$$
\mathcal{L}: \quad x_{1}=d \Omega^{-2}, \quad x_{2}=0, \quad x_{3} \text { is arbitrary, }
$$

parallel to the $O x_{3}$ axis and passing through the point $\left(d \Omega^{-2}, 0,0\right)^{T}$. At the intersection with the cavity $\mathcal{C}$, there is a segment $\left(x_{1 \star}, 0, x_{3}\right)^{T}$, where $-x_{3 \star} \leqslant x_{3} \leqslant x_{3 \star}$. It relates to the $I_{L}$ family of libration points. The RPM is a ball $\mathcal{C}$. 


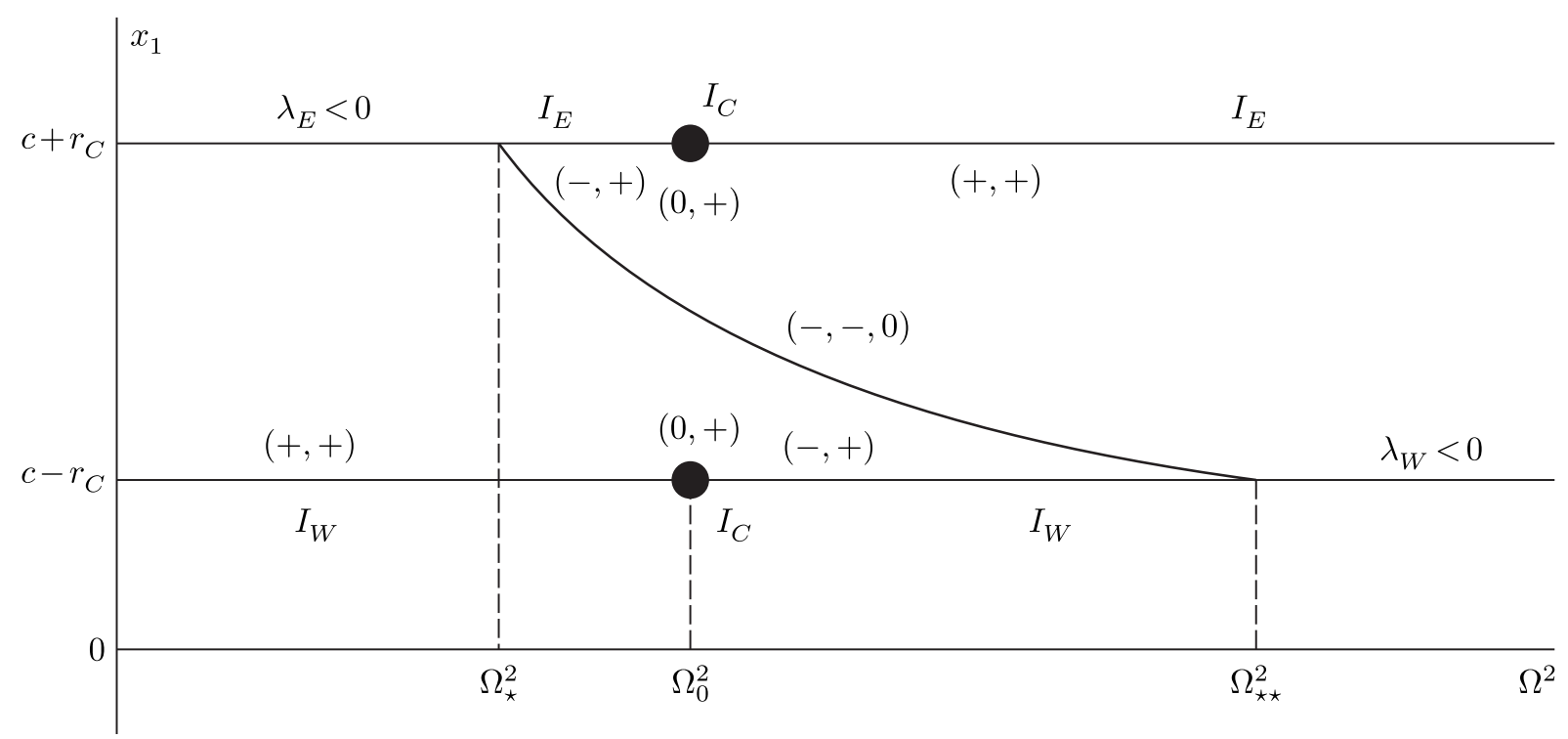

Fig. 3. Bifurcation diagram: the case $c>r_{C}$

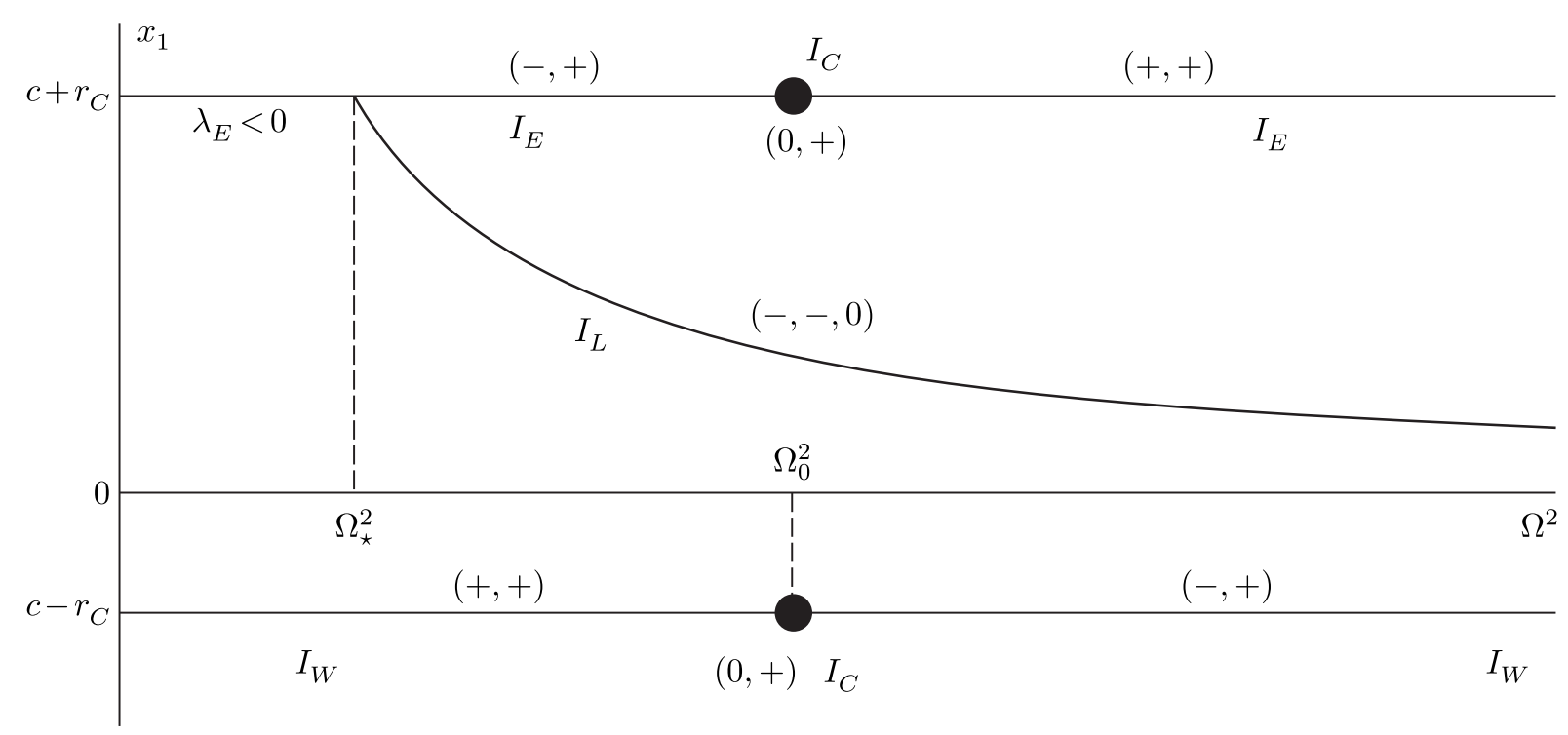

Fig. 4. Bifurcation diagram: the case $c \leqslant r_{C}$

Finally, for $d^{2}-2 \Omega^{2} h>0$, the inequality determines a region $\mathcal{S}$ containing a straight circular cylinder $\partial \mathcal{S}$ with radius $R=\Omega^{-2} \sqrt{d^{2}-2 \Omega^{2} h}$ and the axis coinciding with the straight line $\mathcal{L}$, and the exterior $\mathcal{S} \backslash \partial \mathcal{S}$ of this cylinder.

If the equality

$$
\left(c-r_{C}-d \Omega^{-2}\right)^{2}=\Omega^{-4}\left(d^{2}-2 \Omega^{2} h\right) \quad \Longleftrightarrow \quad h_{W}(\Omega)=\frac{1}{2}\left(c-r_{C}\right)\left(2 d-\left(c-r_{C}\right) \Omega^{2}\right)
$$

is satisfied, the surface of the cylinder $\partial \mathcal{S}$ touches the cavity at the point $W=\left(c-r_{C}, 0,0\right)$. 


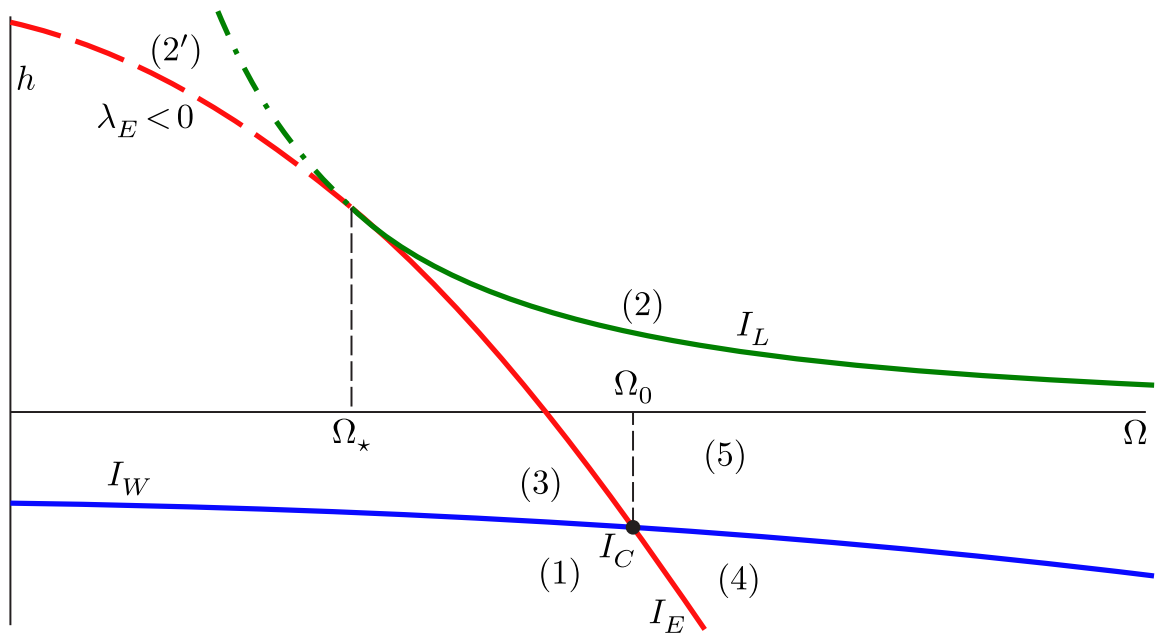

Fig. 5. Bifurcation diagrams on the plane $(\Omega, h)$ for $c<r_{C}$

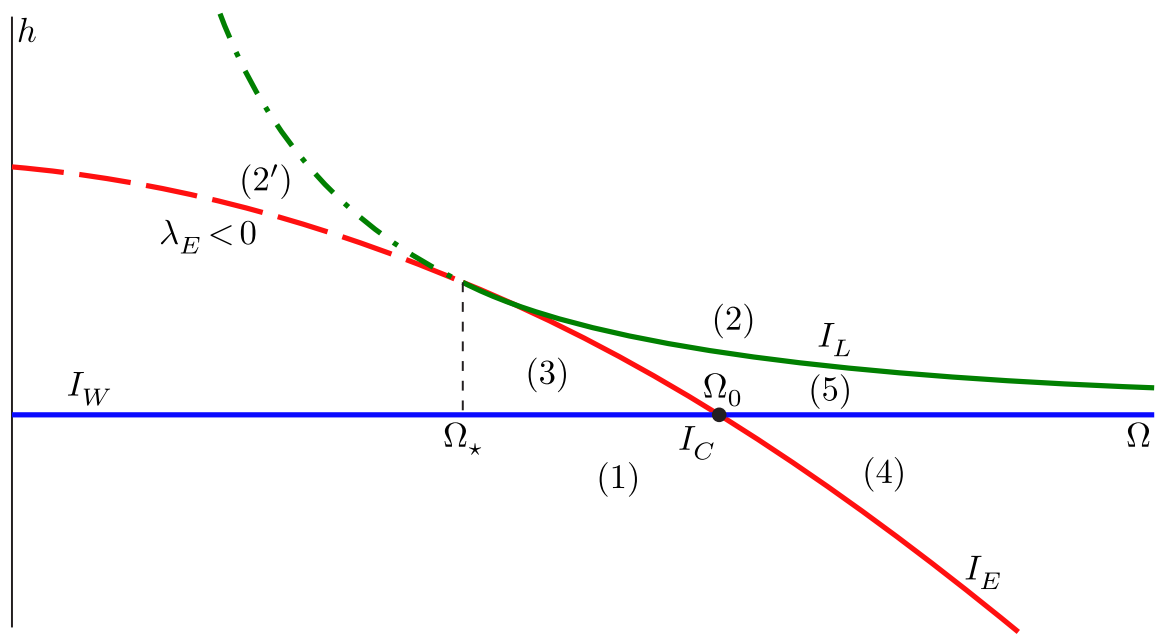

Fig. 6. Bifurcation diagrams on the plane $(\Omega, h)$ for $c=r_{C}$

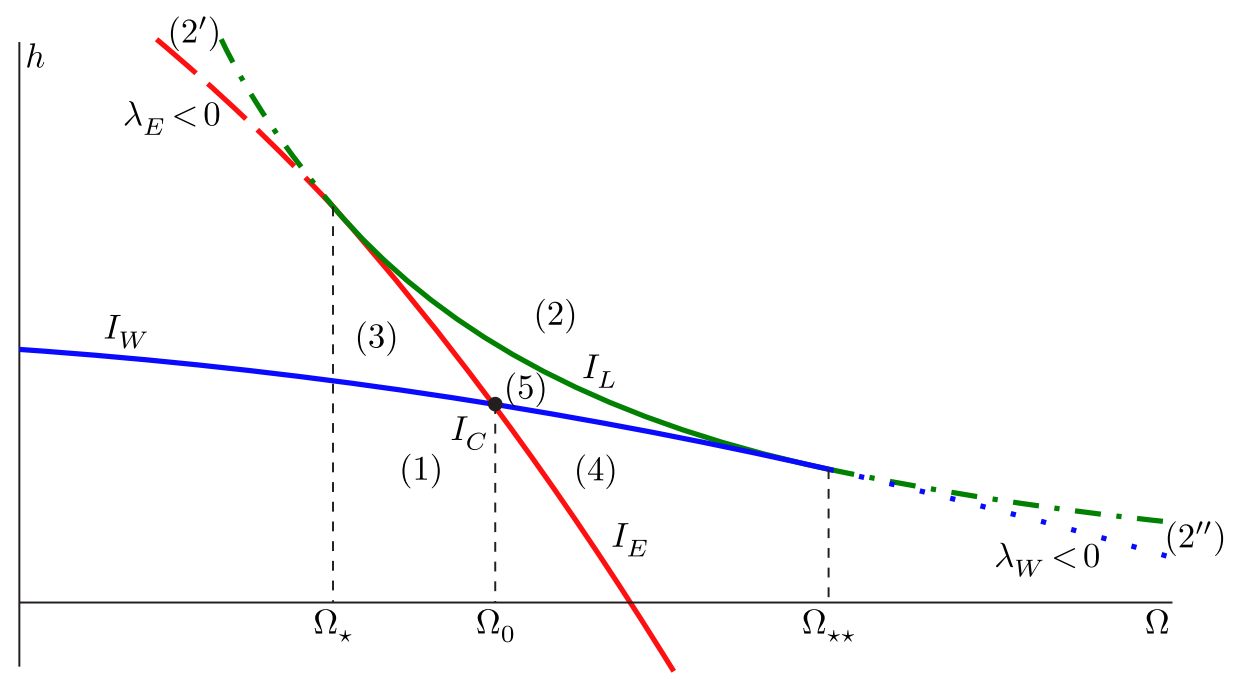

Fig. 7. Bifurcation diagrams on the plane $(\Omega, h)$ for $c>r_{C}$ 
In Figs. $5-7$, the corresponding curve is shown in blue, it relates to the $I_{W}$ family of libration points. The dotted line shows the part of the curve that does not correspond to real motions due to the negative reaction of constraint.

If the equality

$$
\left(c+r_{C}-d \Omega^{-2}\right)^{2}=\Omega^{-4}\left(d^{2}-2 \Omega^{2} h\right) \quad \Longleftrightarrow \quad h_{E}(\Omega)=\frac{1}{2}\left(c+r_{C}\right)\left(2 d-\left(c+r_{C}\right) \Omega^{2}\right)
$$

is satisfied, then the surface of the cylinder $\partial \mathcal{S}$ touches the cavity at the point $E=\left(c+r_{C}, 0,0\right)$.

In Figs. 5-7, the corresponding curve is shown in red, it relates to the $I_{E}$ family of libration points. The dashed line shows a part of the curve that also does not correspond to real motions due to the negative reaction of constraint.

There is also a solid green curve in each of Figs. 5-7. It corresponds to the $I_{L}$ family of libration points. The radius of the cylinder $\mathcal{S}$ vanishes for points belonging to this curve and its continuation drawn by the dot-dashed line. For points above this curve, there are no restrictions on the RPM from the side of the augmented potential. The only point of intersection of the blue and red curves corresponds to the $I_{C}$ libration points. Its coordinates read $\left(\Omega_{0}, h\left(\Omega_{0}\right)\right)$, where $h\left(\Omega_{0}\right)=\frac{c\left(c^{2}-r_{C}^{2}\right)}{2 d}$. The coordinates of the other two corner points in the bifurcation diagram have the form $\left(\Omega_{\star}, h\left(\Omega_{\star}\right)\right)$ and $\left(\Omega_{\star \star}, h\left(\Omega_{\star \star}\right)\right)$ where $h\left(\Omega_{\star}\right)=\frac{d\left(c+r_{C}\right)}{2}$ and $h\left(\Omega_{\star \star}\right)=$ $=\frac{d\left(c-r_{C}\right)}{2}$, respectively. The point $\left(\Omega_{\star \star}, h\left(\Omega_{\star \star}\right)\right)$ exists if the inequality $c>r_{C}$ is satisfied. If $c \rightarrow r_{C}$, then this point tends to infinity (Fig. 6). Finally, this point does not exist if the inequality $c<r_{C}$ holds true (Fig. 7).

If the double inequality

$$
c-r_{C} \leqslant d \Omega^{-2} \leqslant c+r_{C}
$$

is satisfied, the axis of the cylinder $\mathcal{S}$ intersects or touches the cavity $\mathcal{C}$. This inequality reduces to inequality (5.3) for $c>r_{C}$ and to inequality (5.2) for $c \leqslant r_{C}$.
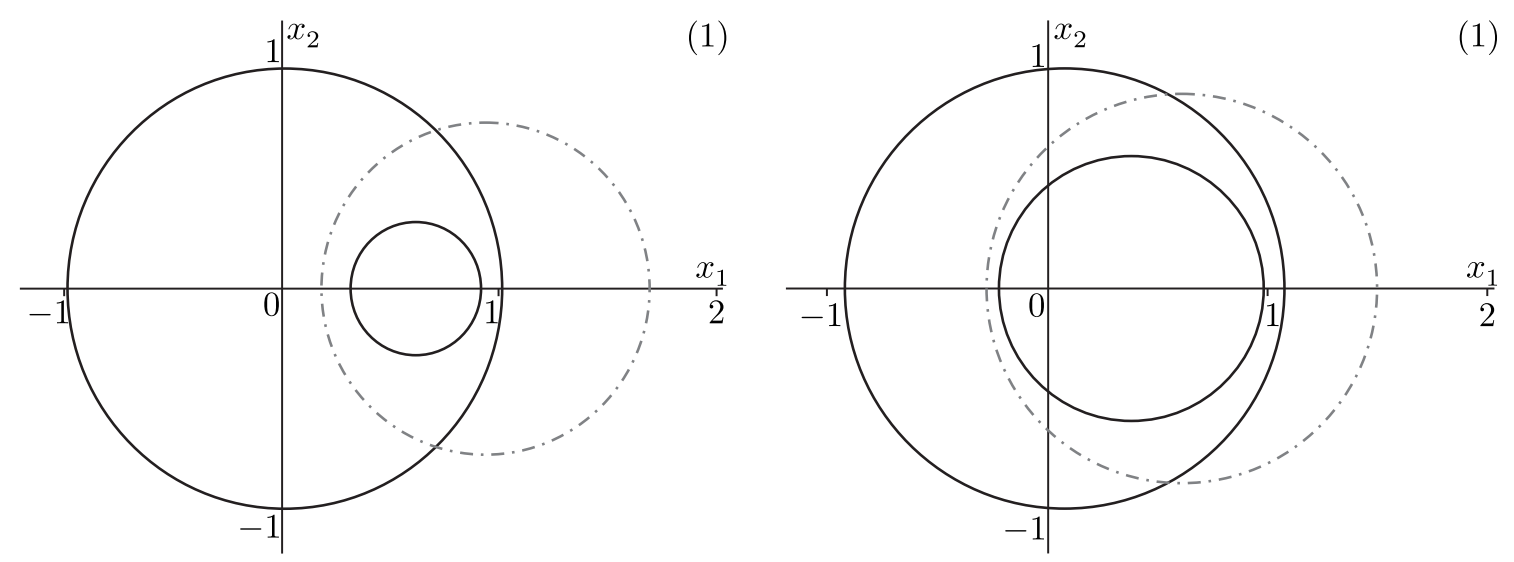

Fig. 8. Projections of RPM onto the plane $O x_{1} x_{2}$ for $c>r_{C}$ (left) and for $c<r_{C}$ (right) in the case of region (1)

Figures 8-12 show the projections of the RPM onto the plane $O x_{1} x_{2}$ for $c>r_{C}$ (left) and $c<r_{C}$ (right), respectively. The section of the body $\mathcal{A}$ is represented by a solid line, and the section of the cylinder $\mathcal{S}$ is represented by a gray dashed circle. The realms of possible motion are highlighted in gray. For parameters from domain (1), the RPM is empty. For parameters from domains $(2),\left(2^{\prime}\right)$, and $\left(2^{\prime \prime}\right)$ the RPM is the entire cavity $\mathcal{C}$. For parameters from domain $(3)$ the $\mathrm{RPM}$ is the part of the cavity $\mathcal{C}$ adjacent to the point $W$. On the contrary, for parameters from 

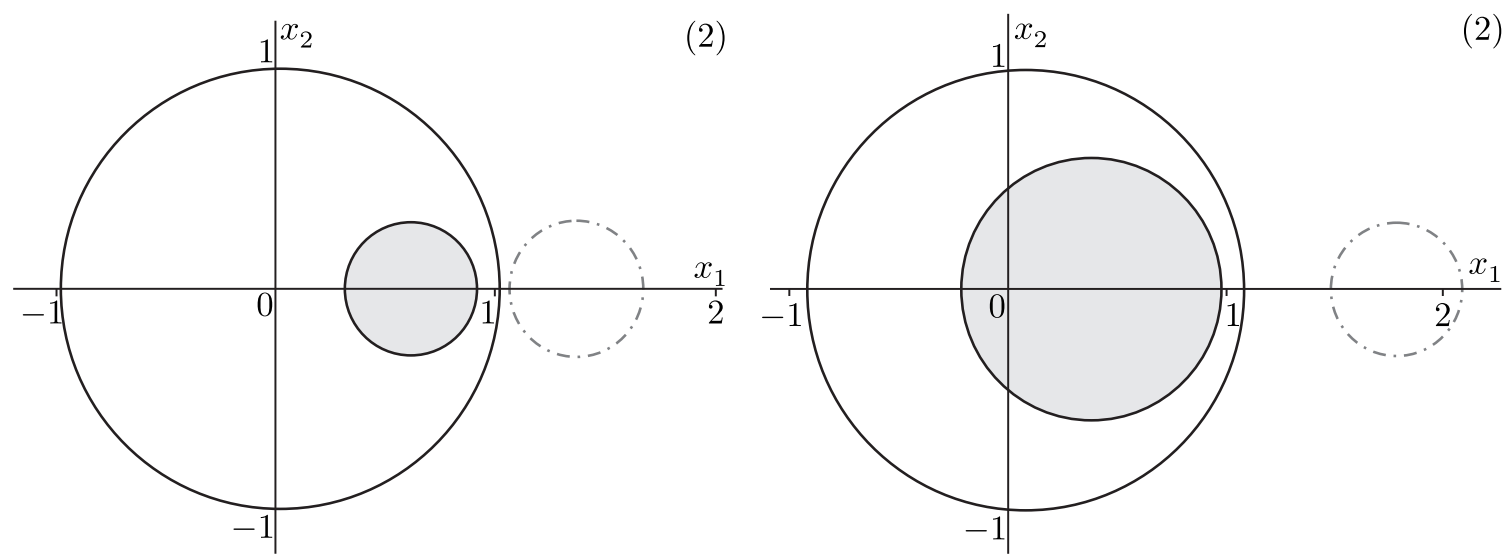

Fig. 9. Projections of RPM onto the plane $O x_{1} x_{2}$ for $c>r_{C}$ (left) and for $c<r_{C}$ (right) in the case of region $(2)$
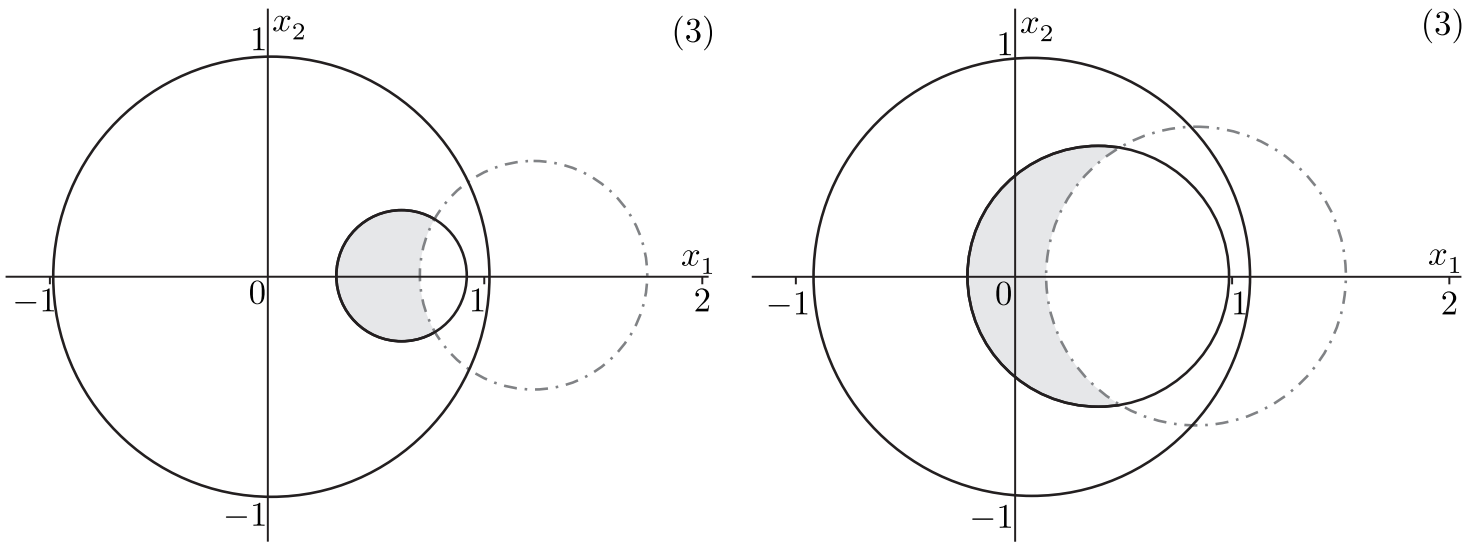

Fig. 10. Projections of RPM onto the plane $O x_{1} x_{2}$ for $c>r_{C}$ (left) and for $c<r_{C}$ (right) in the case of region $(3)$
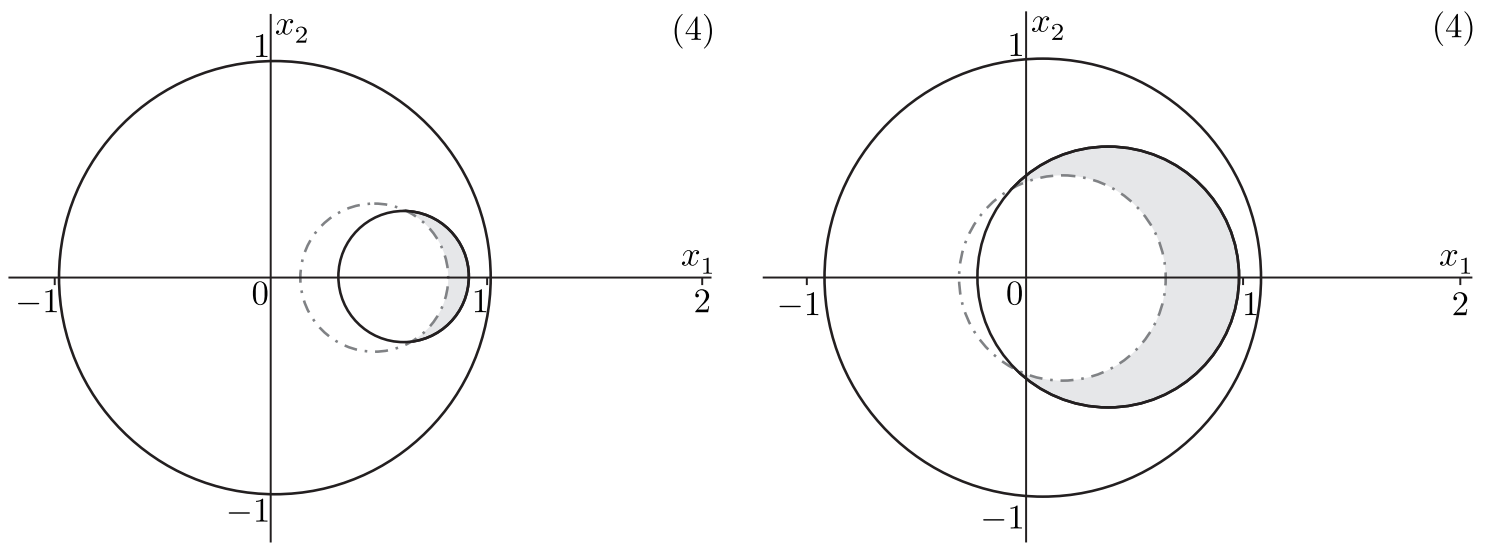

Fig. 11. Projections of RPM onto the plane $O x_{1} x_{2}$ for $c>r_{C}$ (left) and for $c<r_{C}$ (right) in the case of region (4) 

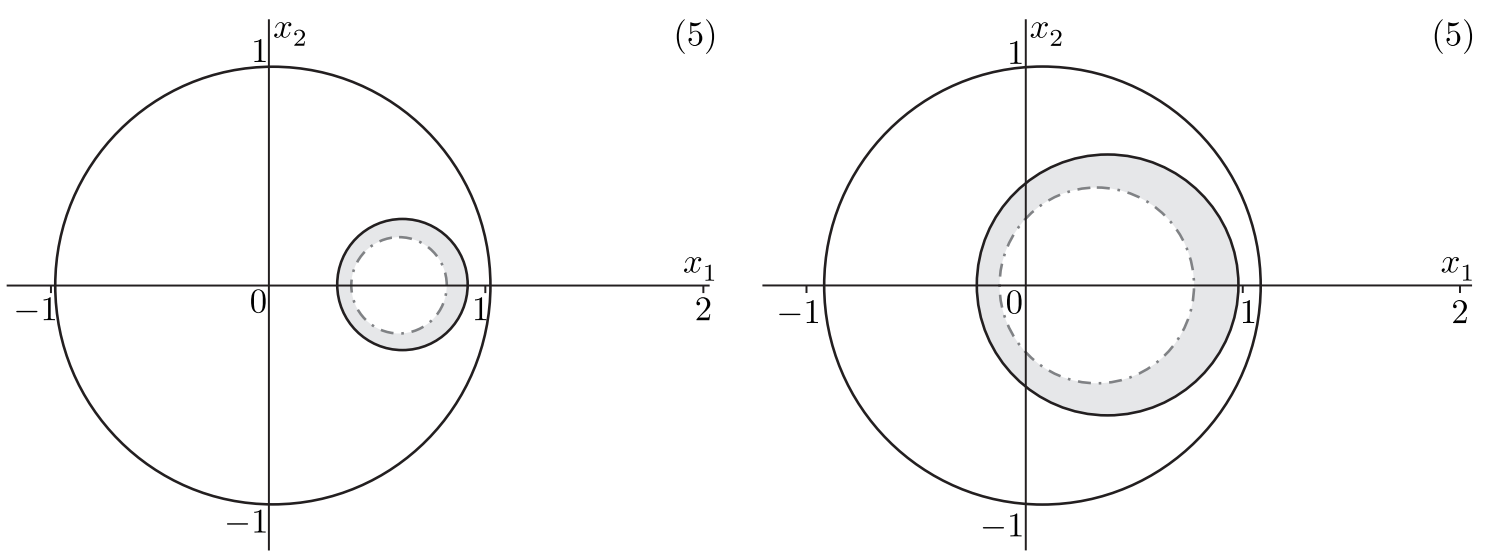

Fig. 12. Projections of RPM onto the plane $O x_{1} x_{2}$ for $c>r_{C}$ (left) and for $c<r_{C}$ (right) in the case of region (5)

domain (4) the RPM is the part of the cavity $\mathcal{C}$ adjacent to the point $E$. Finally, for parameters from domain (5), the RPM is the part of the cavity $\mathcal{C}$, topologically equivalent to the solid torus. It is noteworthy that the realms of possible motion of this type only contain the whole $I_{c}$ family of libration points.

\section{General remarks}

REMARK 5. It is natural to consider a body with a cavity as a generalization of dumbbell-like bodies. The investigation of a dumb-bell as a model of binary asteroids probably dates back to the studies of V. V. Beletsky and A. V. Rodnikov $[14,15]$. When studying the libration points of precessing bodies with a cavity, the results of [16] turns out to be useful.

REMARK 6. If we assume that the walls of the cavity (or the outer surface) are permeable, then a version of the so-called Robe's problem [5] arises. Within this problem, a point (or a body, completely or partially) is submerged inside the attracting medium. This formulation of the problem is of interest from the point of view of cosmology. Permeable gravitational objects are used, e.g., to simulate the motion of a star inside a galaxy (see, e. g., [17]), as well as dust clouds or dust planets [18] (see also [19-21]). Note that in the case where the dimensions of the submerged body are finite, it is necessary to take into account the buoyant force acting on it.

REMARK 7. The mechanics of systems with negative masses (either gravitational, or inertial) is the subject of active study not only in celestial mechanics (see, e. g., [22-25]), but also in theoretical physics (see, e.g., [26]). Objects with cavities are, in our opinion, good examples of systems that often exhibit somewhat unexpected dynamical effects (see, e.g., [27]).

REMARK 8. For rotating gravitating bodies in the presence of dry friction, the relative equilibria become uninsulated both in the case where the point moves along the external surface of the body [28] and in the case of motion along the surface of the cavity existing in it [29].

REMARK 9. The motion of celestial bodies with cavities is also of methodological interest. For example, the problem of the orbital motion of a body with a cavity makes it possible to clarify the physical background of events described in the fascinating, instructive and in many ways prophetic children's book "Dunno on the Moon" written by N. Nosov [30] ${ }^{1}$.

The authors thank the reviewers for the friendly constructive criticism.

\footnotetext{
${ }^{1}$ For the English-speaking reader, generally speaking, only the translation of the first book [31] from the trilogy about Dunno is available. One can read about the cited third book of the trilogy, e. g., on the website https://en.wikipedia.org/wiki/Dunno_on_the_Moon
} 


\section{References}

[1] Scheeres, D. J., Orbital Motion in Strongly Perturbed Environments: Applications to Asteroid, Comet and Planetary Satellite Orbiters, Berlin: Springer, 2012.

[2] Ivashkin, V. V., Stikhno, C. A., and Guo, P., On the Structure of the Set of Probable Earth-Impact Trajectories for the Asteroid Apophis in 2036, Dokl. Phys., 2017, vol. 62, no. 8, pp. 387-391; see also: Dokl. Akad. Nauk, 2017, vol. 475, no. 4, pp. 389-394.

[3] Ivashkin, V. V. and Lang, A., Analysis of the Orbital Motion of the Asteroid Apophis' Satellite, Cosmic Research, 2017, vol. 55, no. 4, pp. 253-262; see also: Kosmicheskie Issledovaniya, 2017, vol. 55 , no. 4 , pp. 268-277.

[4] Ivashkin, V. V., Krylov, I. V., and Lan, A., Optimum Trajectories for Spacecraft Mission to Asteroid Apophis with a Return to the Earth, Solar Syst. Res., 2013, vol. 47, no. 4, pp. 334-345; see also: Astronom. Vestn., 2013, vol. 47, no. 4, pp. 361-372.

[5] Robe, H. A. G., A New Kind of 3-Body Problem, Celestial Mech. Dynam. Astronom., 1977, vol. 16, no. 3, pp. 343-351.

[6] Gendenstein, L. E., Kirik, L. A., and Gelfgat, I. M., Solution of Key Problems in Physics for Basic School: Grades 7-9, Moscow: Ileksa, 2016 (Russian).

[7] Chetaev, N. G., Theoretical Mechanics, Berlin: Springer, 1989.

[8] Routh, E. J., A Treatise on Stability of a Given State of Motion, London: McMillan, 1877.

[9] Routh, E. J., The Advanced Part of a Treatise on the Dynamics of a System of Rigid Bodies: Being Part II of a Treatise on the Whole Subject, 6th ed., New York: Dover, 1955 (reprint).

[10] Karapetyan, A. V., The Stability of Steady Motions, Moscow: Editorial URSS, 1998 (Russian).

[11] Rubanovsky, V. N. and Samsonov, V. A., Stability of Stationary Motions in Examples and Problems, Moscow: Nauka, 1988 (Russian).

[12] Ivanov, A.P., On the Stability of Systems with Non-Retaining Constraints, J. Appl. Math. Mech., 1984, vol. 48, no. 5, pp. 523-528; see also: Prikl. Mat. Mekh., 1984, vol. 48, no. 5, pp. 725-732.

[13] Burov, A. A., The Existence and Stability of the Equilibria of Mechanical Systems with Constraints Produced by Large Potential Forces, J. Appl. Math. Mech., 2003, vol. 67, no. 2, pp. 193-200; see also: Prikl. Mat. Mekh., 2003, vol. 67, no. 2, pp. 222-230.

[14] Beletsky, V.V., Generalized Restricted Circular Three-Body Problem As a Model for Dynamics of Binary Asteroids, Cosmic Research, 2007, vol. 45, no. 5, pp. 408-416; see also: Kosmicheskie Issledovaniya, 2007, vol. 45, no. 5, pp. 435-442.

[15] Beletskii, V. V. and Rodnikov, A. V., Stability of Triangle Libration Points in Generalized Restricted Circular Three-Body Problem, Cosmic Research, 2008, vol. 46, no. 1, pp. 40-48; see also: Kosmicheskie Issledovaniya, 2008, vol. 46, no. 1, pp. 42-50.

[16] Munitsyna, M. A., Relative Equilibria of a Point in a Gravity Field of a Symmetrical Rigid Body, in Problems of Investigating the Stability and Stabilization of Motion, S. Ya. Stepanov, A. A. Burov (Eds.), Moscow: A. A. Dorodnitsyn Computing Centre of the Russian Academy of Science, 2009, pp. 3-8 (Russian).

[17] Gasanov, S. A. and Luk'yanov, L. G., The Libration Points for the Motion of a Star inside an Elliptical Galaxy, Astron. Rep., 2002, vol. 46, no. 10, pp. 851-857; see also: Astron. Zh., 2002, vol. 79, no. 10, pp. 944-951.

[18] Avinash, K., Eliasson, B., and Shukla, P. K., Dynamics of Self-Gravitating Dust Clouds and the Formation of Planetesimals, Phys. Lett. A, 2006, vol. 353, no. 2, pp. 105-108.

[19] Plastino, A. R. and Plastino, A., Robe's Restricted Three-Body Problem Revisited, Celest. Mech. Dyn. Astron., 1995, vol. 61. no. 2, pp. 197-206.

[20] Hallan, P. P. and Rana, N., The Existence and Stability of Equilibrium Points in the Robe's Restricted Three-Body Problem, Celest. Mech. Dyn. Astron., 2001, vol. 79, no. 2, pp. 145-155.

[21] Valeriano, L. R., Parametric Stability in Robe's Problem, Regul. Chaotic Dyn., 2016, vol. 21, no. 1, pp. $126-135$.

[22] Celli, M., Homographic Three-Body Motions with Positive and Negative Masses, in Proc. of the Internat. Conf. on Symmetry and Perturbation Theory (SPT'2004, Cala Gonone, Italy, June 2004), 
G. Gaeta, B. Prinari, S. Rauch-Wojciechowski, S. Terracini (Eds.), Singapore: World Sci., 2005, pp. $75-82$.

[23] Celli, M., Sur les mouvements homographiques de $N$ corps associés à des masses de signe quelconque, le cas particulier où la somme des masses est nulle, et une application à la recherche de chorégraphies perverses, PhD Thesis, Université Paris-Diderot, Paris VII, 2005, 63 p.

[24] Celli, M., The Central Configurations of Four Masses $x,-x, y,-y$, J. Differential Equations, 2007, vol. 235, no. 2, pp. 668-682.

[25] Piña, E. and Lonngi, P., Newtonian Few-Body Problem Central Configurations with Gravitational Charges of Both Signs, arXiv:1212.3219 (11 Dec 2012).

[26] Shatskiy, A. A., Novikov, I. D., and Kardashev, N. S., The Kepler Problem and Collisions of Negative Masses, Physics-Uspekhi, 2011, vol. 54, no. 4, pp. 381-385; see also: Uspekhi Fiz. Nauk, 2011, vol. 181, no. 4, pp. 399-403.

[27] Voronov, V., Gravitational "Repulsion", Quantum, 2009, no. 3, pp. 37-40 (Russian).

[28] Burov, A. A., Kosenko, I. I., and Shalimova, E. S., Relative Equilibria of a Massive Point on a Uniformly Rotating Asteroid, Dokl. Phys., 2017, vol. 62, no. 7, pp. 359-362; see also: Dokl. Akad. Nauk, 2017, vol. 475, no. 3, pp. 269-272.

[29] Burov, A.A., Nikonov, V.I., and Shalimova, E. S., Motion of a Massive Point along the Surface of a Homogeneous Ball with a Spherical Cavity, Prikl. Mat. Mekh., 2021, vol. 85, no. 4, pp. 528-543 (Russian).

[30] Nosov, N. N., Dunno on the Moon, Moscow: Detskaya Literatura, 1965 (Russian).

[31] Nosov, N. N., The Adventures of Dunno and His Friends, Moscow: Progress, 1980. 